\title{
Ciclo de mejora docente en la asignatura afecciones médicas I del grado de Fisioterapia y el doble grado en Fisioterapia y Ciencias del Deporte
}

Mạ ANGELES VÁZQUEZ GÁMEZ

Departamento de Medicina. Facultad de Medicina Universidad de Sevilla mavazquez@us.es

ORCID: http://orcid.org/0000-0001-7689-6999

D.O.I.: http://dx.doi.org/10.12795/JDU.2018.i01.90

Pp.: 1606-1621

\section{Resumen:}

En este ciclo de mejora se ha trabajado partiendo de un caso clínico sobre el que han versado todas las cuestiones que conectaban diferentes temas de la asignatura ( 4 temas concretamente, todos ellos relacionados con el infarto agudo de miocardio). Los alumnos han trabajado en pequeños grupos con diferentes actividades de contraste, dependiendo de la materia a trabajar (videos, cuestionarios, encuestas de opinión), todas ellas relacionadas con el caso clínico inicial y pensadas para favorecer esta metodología de aprendizaje, el debate entre ellos y la corrección de errores en el aula. La participación e implicación del alumnado ha sido alta, con una tasa de abandono prácticamente nula. Sobre la valoración de la progresión del aprendizaje 
de los alumnos, esta comunicación muestra la importancia de llevar a cabo una metodología centrada en el proceso de desarrollo de las escaleras de aprendizaje. Pequeños errores en el diseño, pueden subestimar esta herramienta de evaluación tan útil.

Palabras clave: Afecciones-Médicas I, Fisioterapia, Docencia universitaria, Experimentación docente universitaria, Aprendizaje basado en casos

\section{Breve descripción del contexto:}

Este ciclo de mejora docente (CMD) se ha aplicado en 2 grupos de alumnos de 2 o curso del Grado de Fisioterapia y del doble Grado en Fisioterapia y Ciencias del Deporte, en la asignatura Afecciones Médicas I que se imparte en el primer cuatrimestre. El promedio de alumnos en cada grupo ha sido de 34. El ciclo se ha llevado a cabo en 6 horas de docencia, en días consecutivos en cada una de los grupos (1 hora/día).

\section{Diseño previo del ciclo de mejora docente:}

Hemos trabajado este CMD partiendo de un caso clínico sobre el que han versado todas las cuestiones que conectaban diferentes temas de la asignatura y utilizando distintas actividades de contraste. Hemos construido un mapa de contenidos (fig. 1), categorizándolos en conceptuales, procedimentales y actitudinales. Hemos elaborado 5 preguntas fundamentales que permiten integrar todos los contenidos que queremos trabajar en este CMD. Un video de un paciente con Infarto Agudo de Miocardio (IAM), ha sido el núcleo central, y a partir de él, hemos trabajado los diferentes apartados relacionados con esta patología: factores de riesgo, fisiopatología, RCP, déficit funcionales, tratamiento y prevención.

Jornadas de Formación e Innovación Docente del Profesorado I № 1 (2018) Esta obra se distribuye con la licencia Creative Commons 


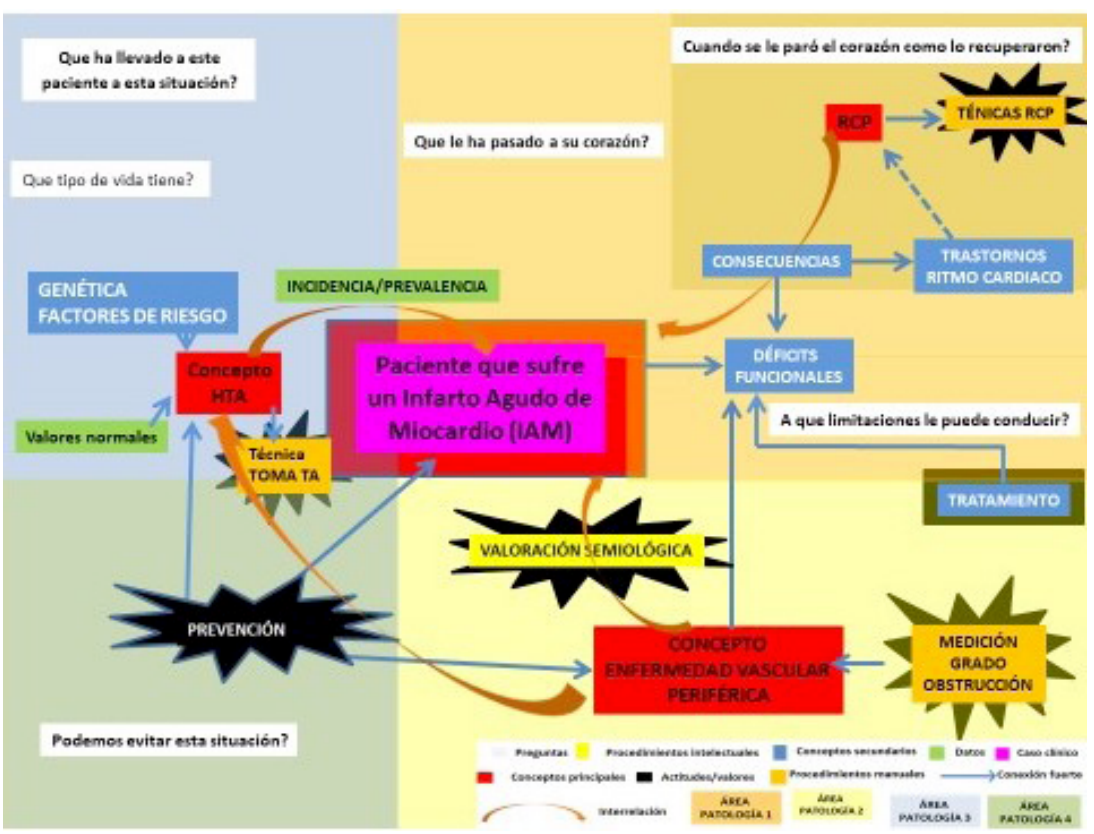

Figura 1. Mapa de contenidos y problemas:

Nuestro modelo metodológico se basa en lo descrito por Escribano y cols. (2008), en el que partimos de un caso o problema sobre el que trabajan los alumnos en pequeños grupos de forma colaborativa, expresando sus ideas y sobre el que se proponen diferentes actividades de contraste (técnicas manuales, videos, encuestas, exposición y debate, trabajos en grupo, etc.), interrelacionándose todos los pasos del modelo. Se han ajustado los tiempos necesarios para cada actividad. La figura 2 muestra el modelo metodológico que hemos seguido para aplicar este CMD. Como se puede observar, solemos dar información previa a la asistencia al aula, para que el alumno traiga una mínima información y agilicemos la clase.

Jornadas de Formación e Innovación Docente del Profesorado I № 1(2018) Reconocimiento-NoComercial-SinObraDerivada Internacional (CC BY-NC-ND 4.0.) 
AC: Actividades de contraste previas a la asistencia al aula.

$\mathrm{ACD}$ : Actividades de contraste docentes (Indicaciones, explicaciones, etc)

$\mathrm{C} / \mathrm{P} / \mathrm{Vp}$ : Caso/problema/video problema

LA: Ideas de los alumnos (trabajos en grupo pequeño, exposición y debate entre grupos, etc)

ACE: Actividades de contraste basadas en experimentos

\section{$5^{\prime}$}

$10^{\prime}$

Figura 2. Modelo metodológico posible

Tabla 3: Secuencia de actividades del ciclo de mejora

En las siguientes tablas se incluyen las fichas de actividades partiendo de los problemas seleccionados.

\begin{tabular}{|c|c|c|}
\hline № ACTIVIDAD & DESCRIPCIÓN ACTIVIDAD DIA 1 & TIEMPO \\
\hline$A C$ & $\begin{array}{c}\text { Actividades de contraste: Previa a la } \\
\text { clase, se suben a plataforma los } 4 \text { te- } \\
\text { mas a tratar en el ciclo, para que el } \\
\text { alumno repase la primera parte de es- } \\
\text { tos temas (recuerdo anatomofisioló- } \\
\text { gico del corazón y el árbol vascular } \\
\text { arterial. } \\
\text { Se sube un video sobre un paciente } \\
\text { con IAM, y un cuestionario anónimo } \\
\text { con } 5 \text { preguntas relacionadas con el } \\
\text { tema que deberán traer contestadas } \\
\text { individualmente a clase }\end{array}$ & Sin tiempo \\
\hline$A C D$ & $\begin{array}{c}\text { Exploro brevemente, y de forma ver- } \\
\text { bal, que saben del tema que vamos a } \\
\text { tratar en clase. Explico el concepto de } \\
\text { cardiopatía isquémica. } \\
\text { Lanzo una de las preguntas que les } \\
\text { hice en la encuesta: "Que le ha pasado } \\
\text { a su corazón?" para que la trabajen. }\end{array}$ & 5 minutos \\
\hline
\end{tabular}

Jornadas de Formación e Innovación Docente del Profesorado I № 1 (2018) Esta obra se distribuye con la licencia Creative Commons 


\begin{tabular}{|c|c|c|}
\hline IA (TGP) & $\begin{array}{l}\text { En el aula, les entrego una batería de } \\
\text { preguntas sobre la patología que va- } \\
\text { mos a trabajar (IAM), y les explico } \\
\text { cómo trabajar. Pueden consultar la in- } \\
\text { formación subida a plataforma (video, } \\
\text { temas de clase, e internet. }\end{array}$ & 30 minutos \\
\hline $\mathrm{IA}(\mathrm{E}+\mathrm{D})$ & $\begin{array}{l}\text { Un grupo elegido por sorteo expone, y } \\
\text { se genera debate entre los grupo }\end{array}$ & 20 minutos \\
\hline Medios: & $\begin{array}{l}\text { Video, ordenador, proyector, papel, } \\
\text { plataforma virtual Papel y boligrafo }\end{array}$ & \\
\hline
\end{tabular}

\begin{tabular}{|c|c|c|}
\hline NNo ACTIVIDAD & DESCRIPCIÓN ACTIVIDAD DIA 2 & TIEMPO \\
\hline$A C D$ & $\begin{array}{l}\text { Lanzo dos de las preguntas que les hice } \\
\text { en la plataforma: "Que ha llevado a } \\
\text { este paciente a esta situación?" y "Qué } \\
\text { tipo de vida tiene?". }\end{array}$ & 5 minutos \\
\hline IA (TGP) & $\begin{array}{l}\text { En el aula, a partir del video que pue- } \\
\text { den consultar, los temas de clase y un } \\
\text { artículo subido a plataforma, tienen } \\
\text { que responder una batería de pregun- } \\
\text { tas sobre factores de riesgo de la enfer- } \\
\text { medad cardiovascular, centradas en las } \\
\text { dos preguntas anteriores. Nos centra- } \\
\text { remos sobre todo en la HTA. Les explico } \\
\text { cómo trabajar. }\end{array}$ & 30 minutos \\
\hline $\mathrm{I} A(E+D)$ & $\begin{array}{c}\text { Exposición de lo trabajado por } 1 \text { grupo } \\
\text { elegido por sorteo y debate con el resto } \\
\text { de grupos }\end{array}$ & 10 minutos \\
\hline ACE & $\begin{array}{c}\text { Actividades de contraste basadas en un } \\
\text { experimento: tomar la Tensión arterial } \\
\text { por grupos, en situación de reposo y } \\
\text { tras actividad física }\end{array}$ & 10 minutos \\
\hline Medios: & $\begin{array}{l}\text { Video, ordenador, proyector, papel, pla- } \\
\text { taforma virtual, manguito de presión, } \\
\text { fonendoscopio }\end{array}$ & \\
\hline № ACTIVIDAD & DESCRIPCIÓN ACTIVIDAD DIA 3 & TIEMPO \\
\hline
\end{tabular}

Jornadas de Formación e Innovación Docente del Profesorado | № 1 (2018) Esta obra se distribuye con la licencia Creative Commons Reconocimiento-NoComercial-SinObraDerivada Internacional (CC BY-NC-ND 4.0.) 


\begin{tabular}{|c|c|c|}
\hline$A C D$ & $\begin{array}{c}\text { Puntualizo los datos de interés vistos } \\
\text { en la primera y segunda, clases. Vol- } \\
\text { vemos a los factores de riesgo: arte- } \\
\text { riopatía periférica por ateroesclerosis. } \\
\text { Explico que es la arteriopatía perifé- } \\
\text { rica y la incidencia y prevalencia del } \\
\text { proceso. }\end{array}$ & 10 minutos \\
\hline C & $\begin{array}{l}\text { Reparto caso clínico para trabajar } \\
\text { (mismo caso del vídeo pero centrado } \\
\text { en el problema de la arteriopatía. }\end{array}$ & 5 minutos \\
\hline IA (TGP) & $\begin{array}{c}\text { Trabajan en grupos pequeños la semio- } \\
\text { logía (síntomas y signos) y las pruebas } \\
\text { complementarias utilizadas, para valo- } \\
\text { rar la arteriopatía. }\end{array}$ & 25 minutos \\
\hline ACE-B-1 & Video medición índice tobillo/brazo. & 2 minutos \\
\hline ACE-B-2 & $\begin{array}{l}\text { Se miden dicho índice en los grupos y } \\
\text { determinan su grado de afectación en } \\
\text { la clasificación clínica }\end{array}$ & 10 minutos \\
\hline Medios: & $\begin{array}{l}\text { Video, ordenador, proyector, papel, pla- } \\
\text { taforma virtual, manguito de presión, } \\
\text { fonendoscopio, doppler. }\end{array}$ & \\
\hline NNo ACTIVIDAD & DESCRIPCIÓN ACTIVIDAD DIA 4 & TIEMPO \\
\hline ACD & $\begin{array}{l}\text { Resumen de lo visto hasta el momento. } \\
\text { Retomar el video en complicaciones del } \\
\text { IAM a partir de la parada cardiorespi- } \\
\text { ratoria. Entrego cuestionario a rellenar. } \\
\text { Lanzo otra pregunta del cuestionario: } \\
\text { "Cuando se le paró el corazón como lo } \\
\text { recuperaron?" }\end{array}$ & 5 minutos \\
\hline C-vídeo RCP & $\begin{array}{l}\text { Ven el vídeo sobre cómo se actúa en la } \\
\text { Parada cardiorespiratoria }\end{array}$ & 7 minutos \\
\hline IA (TGP) & $\begin{array}{c}\text { Contestar a las preguntas de la en- } \\
\text { cuesta sobre la RCP. }\end{array}$ & 23 minutos \\
\hline $\mathrm{I}(\mathrm{E}+\mathrm{D})$ & $\begin{array}{l}\text { Expondrá un grupo elegido por sorteo. } \\
\text { Se debatirá entre todos los grupos. }\end{array}$ & 10 minutos \\
\hline ACE & Ver vídeo de DEA. & 2 minutos \\
\hline ACE & $\begin{array}{l}\text { Buscar el Desfibrilador Externo Auto- } \\
\text { mático (DEA) que está en la Facultad. } \\
\text { (tienen una práctica sobre esto) }\end{array}$ & 8 minutos \\
\hline Medios: & Videos youtube. papel, bolígrafo, DEA & \\
\hline
\end{tabular}

Jornadas de Formación e Innovación Docente del Profesorado | № 1 (2018) Esta obra se distribuye con la licencia Creative Commons 


\begin{tabular}{|c|c|c|}
\hline NNo ACTIVIDAD & DESCRIPCIÓN ACTIVIDAD DIA 5 & TIEMPO \\
\hline$A C D$ & $\begin{array}{l}\text { Resumen de lo visto en clases anterio- } \\
\text { res. Conexión entre los temas vistos. Ex- } \\
\text { plico que actividad vamos a realizar } \\
\text { hoy. Reparto post-it }\end{array}$ & 5 minutos \\
\hline IA(TGP) & $\begin{array}{c}\text { Trabajar que le sugiere a cada miembro } \\
\text { del grupo el IAM. Rellenar un post-it } \\
\text { con una idea de lo que les sugiere el } \\
\text { IAM y lo van pegando en un panel. Cla- } \\
\text { sificarlas y aunarlas generando las } 3 \\
\text { ideas más sugerentes entre todas de } \\
\text { cada grupo }\end{array}$ & 10 minutos \\
\hline IA-I (TFA) & $\begin{array}{c}\text { Recogida de información. Salir a la ca- } \\
\text { lle y preguntar cada grupo a } 5 \text { perso- } \\
\text { nas que les preocupa del IAM. Rellenar } \\
\text { post-it }\end{array}$ & 25 minutos \\
\hline IA (TGP) & $\begin{array}{l}\text { En el aula poner en común ideas de } \\
\text { alumnos y de la población. Ver necesi- } \\
\text { dades. Plantear objetivos. Lo que no de } \\
\text { tiempo lo terminan en casa y traerlo al } \\
\text { aula a la siguiente clase. }\end{array}$ & 15 minutos \\
\hline & $\begin{array}{l}\text { Post-it de colores, panel, papel, } \\
\text { boligrafo. }\end{array}$ & \\
\hline № ACTIVIDAD & Secuencia de actividades del CMD día 6 & TIEMPO \\
\hline$A C D$ & $\begin{array}{l}\text { Resumo las ideas principales de todo } \\
\text { lo visto hasta el momento. Reparto artí- } \\
\text { culo para trabajar en grupo. }\end{array}$ & 5 minutos \\
\hline IA (TGP) & $\begin{array}{c}\text { Trabajo en grupo sobre la valoración } \\
\text { del paciente (objetivos de tratamiento) } \\
\text { para comenzar con el tratamiento de } \\
\text { Fisioterapia. (lo tratan más profunda- } \\
\text { mente en otra asignatura) }\end{array}$ & 20 minutos \\
\hline$I A(E+D)$ & $\begin{array}{c}\text { Un grupo por sorteo expone, y se ge- } \\
\text { nera debate entre los grupo }\end{array}$ & 20 minutos \\
\hline \multirow[t]{2}{*}{ ACE } & $\begin{array}{c}\text { Juego en grupo, con preguntas sobre } \\
\text { los temas tratados en este ciclo de me- } \\
\text { jora docente (QUIZLET final) }\end{array}$ & 10 minutos \\
\hline & Móvil, ordenador, internet & 5 minutos \\
\hline
\end{tabular}

Abreviaturas de las secuencias de actividades: AC: Actividades de contraste previas a la asistencia al aula. ACD: Actividades de contraste docentes (indicaciones, explicaciones, etc). C/P/Vp: Caso/problema/video problema. 
IA: Ideas de los alumnos. TGP: trabajo en grupo pequeño. ACE: Actividades de contraste basadas en experimentos. E+D: Exposición y debate. TFA: Trabajo fuera del aula.

Basándonos en lo descrito por Porlán (2017), al inicio y al final del ciclo, a todos los alumnos se les se les pasó un cuestionario con 5 preguntas consideradas clave, sobre las que se irán desarrollando en clase con los diferentes contenidos incluidos en el mapa, para conocer de dónde partíamos, y poder valorar tras la implantación del CMD, la evolución del aprendizaje en los alumnos. Las encuestas fueron anónimas, identificadas por un lema elegido por el alumno.

Preguntas del Cuestionario:

1. ¿Qué ha llevado a este paciente a esta situación?

2. ¿Qué tipo de vida tiene?

3. ¿Qué le ha pasado a su corazón?

4. ¿A qué limitaciones le puede conducir?

5. ¿Cuándo se le paró el corazón como le recuperaron?

\section{Aplicación del CMD}

El ciclo de mejora se realiza en 2 aulas con una participación de la totalidad de los alumnos que asisten a clase. (una media de 34 alumnos en cada grupo) durante todo el CMD. Mi objetivo fundamental es que el alumno aprenda a integrar los contenidos de diferentes temas, aproximándose a la realidad de la clínica. Antes de instaurar este ciclo, estos temas se han impartido siempre de forma aislada, y aunque se les intentaba relacionar las materias de forma verbal en clase, no surten los resultados deseados. He optado por tanto, por dinamizar mucho más la clase con diferentes actividades que lleven a converger todos los conocimientos en un mismo caso clínico. Con ello, intentaré que el alumno genere conexiones entre toda la información recibida y trabajada. 
La dinámica general de trabajo en ambos grupos ha sido similar y la respuesta del alumnado parecida, si bien uno de los grupos ha sido bastante más participativo y curioso que el otro.

Cada día al llegar a clase, empiezo por explicar la dinámica a seguir, les doy trabajos (cuestionarios sobre la materia a tratar ese día) para realizar en el aula y les indico las actividades a realizar, que he detallado previamente en la secuencia de actividades previa. , Se distribuyen en 8 grupos para realizar las tareas. Los alumnos trabajan muy bien en equipo, participando activamente y sin incidencias. Los primeros días tardaron bastante en acoplarse y entender cómo afrontar el trabajo colaborativo, pero a medida que pasaban los días, el mecánica de trabajo se agilizó muchísimo. El hecho de trabajar de este modo ha facilitado la participación y el aprendizaje de un mayor número de alumnos generando una muy buena conexión entre ellos y evitando la individualidad que tanto les asusta.

El núcleo que ha centrado toda la docencia ha sido un vídeo sobre un caso clínico que centra el CMD. En base a este vídeo se han elaborado las diferentes hipótesis de trabajo. Hemos ido abordando, desde esa perspectiva, los distintos objetivos del mapa de contenido elaborado previamente. La experiencia ha sido muy positiva. Esta forma de trabajar ha generado curiosidad en el alumno, haciéndole mucho más participe en su proceso de aprendizaje. La participación ha sido alta y la respuesta a los trabajos demandados muy buena. El $80 \%$ de los alumnos han entregado las tareas en los plazos acordados. Sí observo, cierta resistencia a las lecturas que se les recomiendan fuera del aula (a la mayoría les cuesta leer el tema por adelantado antes de verlo en clase). Compruebo que son mucho más colaboradores cuanto más actividades procedimentales y 
actitudinales se les incluye en el aprendizaje. Mi labor en el aula ha sido conducir el proceso de aprendizaje mediante la observación, la aclaración de dudas y, posteriormente, la evaluación tanto de aquellos trabajos individuales que he solicitado a los alumnos, como del propio CMD. La variedad en las actividades de contraste incluidas en el CMD, abarcando diferentes aspectos del aprendizaje ha atrapado a los alumnos incrementando la asistencia a clase y fomentando su participación. Me ha llamado la atención, cómo el alumno ha ido adaptándose cada vez mejor a la forma de trabajar, agilizando mucho la dinámica del aula y siendo cada vez más rápidos y productivos resolviendo los cuestionarios.

El llevar un diario de clases ha sido muy útil, permitiéndome modificar actividades en función de cómo respondía el aula al trabajo propuesto.

La evaluación de los alumnos la he realizado con las escaleras de aprendizaje, en base a los cuestionarios que rellenaron los alumnos antes y después de realizar el ciclo de mejora. Antes de revisar los resultados, quiero resaltar que la encuesta inicial fue contestada vía mail (no disponía de más horas presenciales para poder realizarlas en el aula), después de ver un video (que podían revisar cuantas veces quisiesen) y contando con cualquier fuente de información (Internet, apuntes, etc.). Esto es importante a tener en cuenta, para entender los resultados obtenidos. La encuesta final por el contrario, se realizó en el aula y sin poder acceder a ningún tipo de información, es decir, con lo que realmente ha captado cada alumno en dicho ciclo. En la fig. 3 se muestran los resultados obtenidos.

Jornadas de Formación e Innovación Docente del Profesorado I № 1 (2018) Esta obra se distribuye con la licencia Creative Commons 

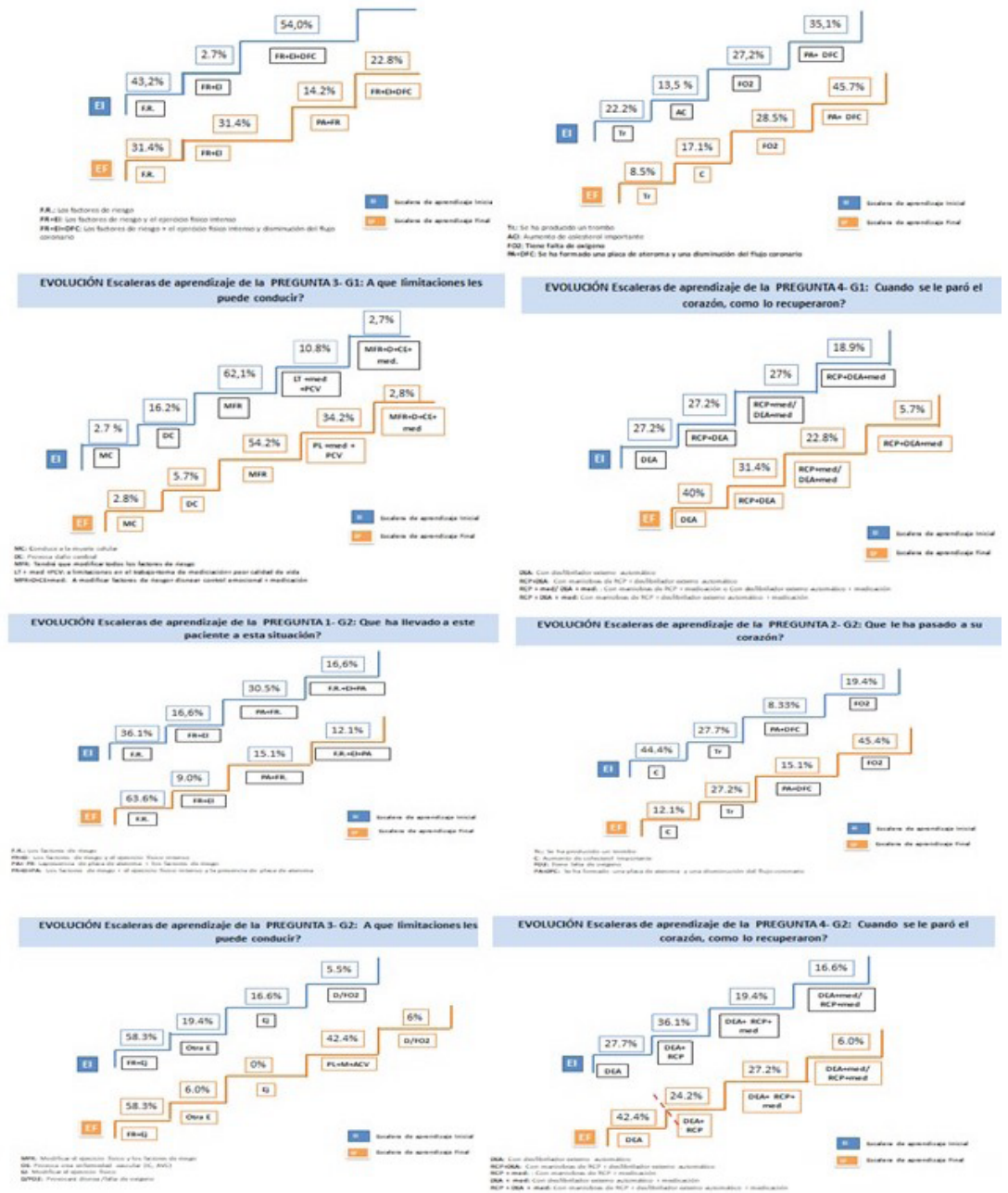

Figura 3. Evolución de las escaleras de aprendizaje en los grupos 1 y 2

Como podemos observar, si no atenemos exclusivamente a la representación gráfica de las escaleras de aprendizaje, no da la sensación que el alumno en general no ha mejorado su aprendizaje, e incluso en ocasiones, parece haber perdido conocimientos, algo totalmente irreal, ya que los trabajos presentados tanto de forma grupal como individual reflejan que los conocimientos están 
adquiridos y mejorados. Cuando he evaluado el comportamiento de las escaleras en alumnos individuales (muestra de 10 alumnos escogidos al azar), he podido observar que el resultado es muy similar a las escalas obtenidas en el grupo.

Tabla 4. Evolución individual de los alumnos (muestra aleatoria)

\begin{tabular}{|c|c|c|c|c|c|c|c|c|c|c|c|c|}
\hline \multicolumn{13}{|c|}{ Evolucion escaleras de aprendizaje } \\
\hline & P-1B & P-1 F & & $\mathrm{P}-2 \mathrm{~B}$ & $\mathrm{P}-2 \mathrm{~F}$ & & P-3B & P-3F & & P-4B & P-4F & \\
\hline Sujeto 1 & B & B & $\leftrightarrow$ & $\mathrm{C}$ & B & $\downarrow$ & $\mathrm{C}$ & $\mathrm{C}$ & $\leftrightarrow$ & $\mathrm{C}$ & B & $\nabla$ \\
\hline Sujeto 2 & $\mathrm{C}$ & $\mathrm{C}$ & $\leftrightarrow$ & B & B & $\leftrightarrow$ & B & B & $\leftrightarrow$ & A & A & $\leftrightarrow$ \\
\hline Sujeto 3 & B & A & $\downarrow$ & $\mathrm{D}$ & D & $\leftrightarrow$ & B & D & $\uparrow$ & $\mathrm{C}$ & B & $\downarrow$ \\
\hline Sujeto 4 & B & A & $\downarrow$ & $\mathrm{D}$ & $\mathrm{D}$ & $\leftrightarrow$ & A & A & $\stackrel{1}{\leftrightarrow}$ & $\mathrm{C}$ & B & $\Downarrow$ \\
\hline Sujeto 5 & A & A & $\leftrightarrow$ & B & $\mathrm{D}$ & $\uparrow$ & A & D & 斥 & $\mathrm{A}$ & D & $\uparrow$ \\
\hline Sujeto 6 & $\mathrm{C}$ & B & $\downarrow$ & $\mathrm{C}$ & D & 个 & $\mathrm{C}$ & $\mathrm{C}$ & $\leftrightarrow$ & $\mathrm{D}$ & D & $\leftrightarrow$ \\
\hline Sujeto 7 & B & B & $\leftrightarrow$ & D & D & $\leftrightarrow$ & B & B & $\leftrightarrow$ & A & A & $\leftrightarrow$ \\
\hline Sujeto 8 & B & A & $\downarrow$ & $\mathrm{C}$ & $\mathrm{C}$ & $\leftrightarrow$ & $\mathrm{C}$ & $\mathrm{C}$ & $\leftrightarrow$ & A & A & $\leftrightarrow$ \\
\hline Sujeto 9 & B & $\mathrm{C}$ & $\uparrow$ & D & D & $\leftrightarrow$ & A & C & $\uparrow$ & $\mathrm{C}$ & D & 个 \\
\hline Sujeto 10 & $\mathrm{C}$ & B & $\downarrow$ & D & D & $\leftrightarrow$ & B & B & $\leftrightarrow$ & $\mathrm{C}$ & B & $\downarrow$ \\
\hline
\end{tabular}

Ante estos resultados, llegamos a las siguientes reflexiones: Hay 2 posibilidades para justificar estos resultados, o bien las preguntas no son suficientemente adecuadas para discernir los conocimientos que tienen los alumnos de base, o bien, al realizar la encuesta inicial vía telemática, pudiendo acceder el alumno a todo tipo de información, han distorsionado los resultados. Esta escalera de aprendizaje inicial no reproduce fielmente los conocimientos de donde partimos. Considero mucho más fiables los resultados obtenidos en la encuesta final, ya que realmente me indican lo que se han quedado los alumnos tras el ciclo de mejora. Por tanto, estas escaleras no son válidas para conocer la evolución de los alumnos después del ciclo de mejora ya que considero que la inicial no muestra resultados reales. Dicha encuesta ha de ser presencial en el aula, sin medios ni recursos donde puedan adquirir información. 


\section{Evaluación del CMD}

El CMD me ha resultado muy productivo y me ha permitido abrir la mente a nuevas posibilidades en el contexto del aprendizaje en el aula. Voy a evaluar por separado los que considero pilares en los que se fundamenta dicho ciclo: a)los alumnos/as, b) los contenidos, c) la metodología y d) la evaluación.

a) En relación a los alumnos he aprendido que es importante conocer los conocimientos que tiene el alumno y de donde vamos a partir. Es decir, conocer los modelos mentales de los estudiantes (Bain, 2007). De ello va a depender el éxito o fracaso de nuestros objetivos docentes. Esta información nos va a ser útil, tanto para adaptarnos de forma más específica al grupo, cómo para hacer la adaptación de alumnos concretos y poder mejorar su aprendizaje. Me parece primordial la empatía e interrelación con el alumnado. La buena armonía en el aula, el no considerar al alumno como alguien inferior al profesor, y el entender no sólo su parcela docente sino también su entorno (familiar, personal, etc), facilitan el acercamiento al alumno, sin temor a que ello genere faltas de respeto ni coleguismo. Esa actitud de proximidad relaja al alumno, lo hace más participativo y le permite expresarse sin miedos ni temor a represalias, como refiere Bain (2007). El familiarizarse con el nombre de los alumnos, crea un clima de confianza en el aula.

b) En cuanto a los contenidos de enseñanza, he comprendido la importancia de planificar por anticipado los objetivos que pretendemos conseguir y cómo se van a abordar (metodología docente), de jerarquizar los contenidos diferenciando los más primordiales de otros menos importantes que tendrá que adquirir el alumno de otra manera 
(lecturas, trabajos, etc.), pero no de forma presencial en el aula. También me he dado cuenta del papel que juegan los mapas de contenido y problemas para conseguir los objetivos antes comentados. El mapa de contenido, como refiere Porlán (2017), nos permite ver de un solo vistazo, y de forma gráfica, lo esencial y lo menos importante, y cómo podemos conectar e interrelacionar los diversos apartados de la materia para que el alumno no vea la formación cómo entes aislados sin ninguna conexión.

Este CMD me ha reafirmado en la importancia de trabajar a partir de casos, sobre todo en áreas de conocimiento como las Ciencias de la Salud. Es importante elaborar, con la ayuda de los mapas de contenido, el caso que permita integrar el mayor número de puntos de interés para el alumno sobre un tema determinado, favoreciendo el aprendizaje y la conexión de los contenidos.

c) El método utilizado, como manifiesta Porlán (2017), juega un papel destacado a la hora de impartir los contenidos, ya que te permite planificar de forma global y conjunta las actividades necesarias para que los alumnos alcancen los conocimientos que nos hemos planteado. Además, el modelo metodológico nos facilita regular los tiempos de trabajo dedicados a cada actividad en función de su complejidad y conocer cómo vas avanzando y cómo has de modificar las siguientes actividades para poder alcanzar las metas previstas.

Otro punto que me he aprendido y me ha parecido muy interesante en este CMD ha sido el construir las preguntas claves encadenadas, redactadas en un lenguaje asequible para el alumno. El utilizar la escritura para responder a 
estas cuestiones, nos permite una reflexión más intensa y cuidada que la que podríamos obtener verbalmente. Como refiere Finkel (2008), en su libro dar clase con la boca cerrada, esta forma de aprendizaje nos permite llegar a conocimientos más elaborados facilitando la consecución de los objetivos propuestos.

d) La evaluación del proceso de aprendizaje es primordial. Es la única manera de saber que está ocurriendo realmente con los alumnos y con el propio método docente, permitiendo hacer cambios para mejorar la docencia (Bain, 2007). Las escaleras de aprendizaje, como método de evaluación de la progresión del conocimiento en los alumnos, es una herramienta muy útil, ya que, como apuntan Porlán (2017), no sólo nos permite conocer tanto la evaluación individual como grupal, sino que también detectar los obstáculos que hay que superar para seguir avanzando en el aprendizaje.

De igual manera es interesante la evaluación del diseño didáctico y del propio docente. Si bien ya existen en la Universidad encuestas de evaluación del profesorado por parte del alumno, es fundamental la autoevaluación del profesor sobre su propio diseño didáctico y su actuación en el aula. Es un proceso de reflexión que nos enriquece y potencia el cambio. Para ello, es fundamental el diario de sesiones del profesor. El registrar toda nuestra actividad en el aula, facilita tremendamente el conocer donde radican los fallos, ver que está funcionando y es útil y reprogramar la docencia haciéndola más acorde a las necesidades de nuestros alumnos. Como refieren Porlán y Martín (1991) es un instrumento útil para la descripción, el análisis y la valoración de la realidad en el aula. 
El aunar todas las actividades en un portafolio, permite tener de forma conjunta todas las actividades realizadas y ayuda a revisar nuestra docencia o el trabajo del alumno en conjunto, incluso pasado mucho tiempo, facilitando el recuerdo (Porlán, 2017).

\section{Conclusiones}

El ciclo de mejora docente aplicado en la asignatura Afecciones Médicas I, ha constituido una herramienta muy útil para mejorar la docencia. El uso de esta metodología ha aumento el interés y la participación del alumno, con mucha mayor implicación en la asignatura. Si bien, la evaluación mediante escaleras de aprendizaje no ha aportado la información deseada en estos grupos porque la metodología no se ha aplicado correctamente, los trabajos realizados por los alumnos durante el CMD, muestran una mejor adquisición de los conocimientos trabajados.

\section{Referencias bibliográficas:}

Bain, K. (2007). Lo que hacen los mejores profesores universitarios. Valencia. Universidad de Valencia

Escribano, A y Del Valle, A. (2008). El aprendizaje basado en problemas. Una propuesta metodológica en Educación superior. Madrid. Narcea.

Finkel, D. (2008). Dar clase con la boca cerrada. Valencia. Universidad de Valencia

Porlán, R. (Coord.) (2017). Enseñanza Universitaria. Cómo mejorarla. Madrid. Morata

Porlán, R. y Martín, J. (1991). El diario del Profesor. Un recurso para la investigación en el aula. Sevilla. Díada. 